\title{
Diferencias en factores de riesgo médico y estilo de vida, entre personas con y sin hipertensión arterial
}

\author{
R. Castillo Ayuso \\ E. M. Escoffié Aguilar \\ J. L. Gutiérrez Pacheco \\ Universidad Autónoma de Yucatán
}

\section{Resumen}

El presente reporte describe las diferencias entre personas con y sin diagnóstico de hipertensión arterial (HTA), en cuanto a factores de riesgo médico (FRM), estilo de vida (EdV) y niveles de ira. Aquí se reportan solamente las diferencias en FRM y EdV, entre personas con y sin hipertensión arterial. Para establecer los FRM y el EdV, se aplicó un cuestionario elaborado para esta investigación y se tomaron medidas antropométricas a una muestra seleccionada intencionalmente, formada por 246 sujetos derechohabientes de un sistema de salud privado del estado de Yucatán, con un rango de edad de 20 a 65 años (=44.30 y $\sigma=10.66)$, de los cuales el 35.4\%, fueron hombres y el 64.6\% fueron mujeres. Se encontró asociación estadísticamente significativas entre el diagnóstico de HTA y la presencia de diabetes $\left(X_{(1)}=14.05, p \leq 0.05\right)$, así como con la presencia de familiar con hipertensión $\left(X_{(1)}=7.73, p \leq 0.05\right)$. Las variables de EdV y los restantes FRM se comportaron de manera similar en los grupos de personas con hipertensión y sin ella. Los resultados sugieren que el EdV funciona como exacerbante de la hipertensión y no como agente causal, y que a pesar de que la HTA y la diabetes están estrechamente relacionadas, no parece existir un nexo de carácter hereditario entre ellas.

\section{Abstract}

The present report studied the differences between people with and without diagnosis of Hypertension (HT), in their Medical Risk Factors (MRF), Lifestyle (LS) and levels of Anger. It was applied the MRF and LS Questionnaire, developed for this research, it was taken anthropometric measures to a purposely selected sample of 246 subjects entitled to a private health system from Yucatan, with an age range of $25-65$ years $(=44.30$ y $\sigma=10.66)$ of which $35.4 \%$ were male and $64.6 \%$ were women. Statistically significant association were found between diagnosis of Hypertension, Diabetes $\left(X_{(1)}^{2}=14.05, p \leq 0.05\right)$ and presence of Family Hypertension $\left(X_{(1)}=7.73, p \leq 0.05\right)$. Variables LS and other MRF behaved similarly in groups of people with Hypertension and without it. It's possible that LS function as Hypertension exacerbating and not as causal agents. Even HT and Diabetes are closing relational; it does not seem to be a hereditary link between them.

Palabras clave: hipertensión arterial, factores de riesgo médico, estilo de vida, diabetes, padecimientos renales. 


\section{Introducción}

Una de las 20 principales causas de muerte a nivel global, así como también, una de las diez principales causas de enfermedades crónicas en todas las regiones del mundo, de acuerdo a la Organización Mundial de la Salud (World Healt Organization, 2004), es la HTA. Dada la alta prevalencia de dicha afección crónica, resulta un problema de salud pública mundial y en nuestro país va en asenso, pues, se consituye en un factor de riesgo cardiovascular reconocido, y es responsable de una gran morbilidad (Hernández, 2005).

La HTA es una enfermedad que se caracteriza por una presión de $\geq 140 \mathrm{~mm} \mathrm{Hg}$ en la presión sistólica y/o una elevación $\geq$ de $90 \mathrm{mmHg}$ en la diastólica (Rosas, 2008). Esta enfermedad impacta de forma importante la salud de la persona y de acuerdo al National Heart Lung and Blood Institute (2008), esta afección por sí sola no produce síntomas, por lo cual resulta de gran relevancia el explorar, describir y relacionar los factores que pudiesen ayudar a su posible pronóstico, para favorecer su prevención.

En una encuesta epidemiológica realizada en México en el año 2000 , se estimó la prevalencia de la hipertensión en un 30.05\% (VelazquezMonroy et al., 2002). Por su parte, de acuerdo a cifras de la Encuesta Nacional de Salud y Nutrición (Instituto Nacional de Salud Pública, 2006), la HTA tiene una prevalencia entre la población mexicana mayor a 20 años, de 15.4\% en 2006 y en la población yucateca se vio una prevalencia de $17.3 \%$. En esta misma encuesta se reportó que en mayores de 20 años, la prevalencia de diabetes mellitus en la población mexicana fue de $7 \%$ en 2006 , mientras que en la yucateca fue de $19.6 \%$.

Entre los FRM relacionados con la hipertensión, que fueron considerados en el presente estudio, se encuentran los padecimientos renales, que son cualquier enfermedad 0 trastorno que afecte al funcionamiento de los riñones; algunos ejemplos son la nefritis crónica y la insuficiencia renal (Silberger, 2010). La diabetes es una enfermedad crónica que aparece cuando el páncreas no produce insulina suficiente 0 cuando el organismo no utiliza eficazmente la insulina que produce (OMS, 2011). Por otra parte, los padecimientos cardiovasculares son cualquier enfermedad o trastorno relacionado con el corazón y los vasos sanguíneos, entre estos se encuentran: la cardiopatía coronaria, enfermedades cerebro vasculares, arteriopatías periféricas, cardiopatía reumática, cardiopatías congénitas, trombosis venosas profundas y embolias pulmonares (OMS, 2011).

De acuerdo a Gasparotto, (2009) los factores de riesgo para la hipertensión son la edad, el sexo, grupo étnico de pertenencia, factores socio económicos, alto consumo de sal, obesidad, consumo de bebidas alcohólicas y sedentarismo; por tanto, resulta relevante estudiar los factores que pueden ser modificables como son el sedentarismo, la obesidad, el tabaquismo, y el consumo de alcohol y sal, entre otros; ya que al ser atendidos de manera oportuna ayudan a la disminución de los índices de morbimortalidad (Montoya y Osorio, 2006). En la presente investigación se evaluaron los estilos de vida mediante autorreporte para conocer en la población el consumo de tabaco, ingesta de alcohol, sedentarismo y consumo de sal.

\section{Método}

\section{Participantes}

Fueron encuestados 246 sujetos pertenecientes a un sistema de salud privado del estado de Yucatán, mediante un muestreo intencional, cuyos criterios de inclusión fueron pertenecer al rango de edad de entre 20 y 65 , que asistieran a consulta externa en dicha institución en el tiempo en el que se realizó la investigación, así como que accedieran a participar de forma voluntaria. 


\section{Instrumentos}

El cuestionario de FRM y de EdV incluyó preguntas demográficas sobre el sexo, la edad, así como sobre el control medicamentoso para la HTA. También evaluó mediante presencia o ausencia los FRM, los factores hereditarios de riesgo médico y las variables del factor $\mathrm{EdV}$. La construcción del instrumento fue teórica con base en los factores reportados frecuentemente asociados a la HTA y la inclusión de los reactivos, así mismo, la redacción se elaboró mediante criterio experto y con la asesoría de dos profesionales de salud (Médicos).

Registro de mediciones antropométricas del área de enfermería. Se empleó dicho registro que incluyó la presión arterial diastólica, presión arterial sistólica, peso, talla e índice de masa corporal (IMC). El registro fue proporcionado por el personal de enfermería posterior al consentimiento informado del sujeto y fue elaborado mediante el registro de los datos provenientes de un baumanómetro analógico, una báscula con estadímetro analógica.

\section{Objetivo del estudio}

Describir las diferencias que existen en los FRM y el EdV entre personas con y sin diagnóstico de HTA.

\section{Preguntas de investigación}

¿Existen diferencias entre personas con y sin diagnóstico de HTA en sus FRM y EdV?

\section{Definición de variables}

Diagnóstico de hipertensión arterial esencial. La HTA se refiere a un aumento en la fuerza de la sangre contra las arterias en el cuerpo, considerada a partir de cifras en presión sistólica y diastólica iguales o mayores a 140/90 mmHg. Para propósitos de la presente investigación se empleó el diagnóstico de hipertensión reportado por los participantes en respuesta al cuestionario de FRM y de $\mathrm{EdV}$, validado con la medición antropométrica realizada por personal médico del centro de salud donde se realizó el estudio.

Las variables denominadas factores de riesgo médico (FRM) se evaluaron de dos formas diferentes: el primer grupo constituido por presión arterial diastólica, presión arterial sistólica, peso talla e índice de masa corporal (IMC), se obtuvieron mediante el registro de mediciones antropométricas del área de enfermería; y el segundo grupo, constituido por las variables de padecimientos cardiovasculares, diabetes y padecimientos renales, las cuales fueron evaluadas mediante auto reporte de diagnósticos preexistentes sobre dichas condiciones en los sujetos mediante el cuestionario de FRM y de EdV.

Las variables denominadas como factores hereditarios de riesgo médico (FHRM) fueron: familiar con hipertensión, familiar con disfunción cardíaca, familiar con diabetes y familiar con disfunción renal; estas fueron evaluadas mediante el autoinforme de la presencia o ausencia de algún familiar de primer grado con el padecimiento evaluado en el cuestionario de FRM y de EdV.

Las variables del factor de estilo de vida que se evaluaron fueron: activación física, consumo de tabaco, ingesta de alcohol y consumo de sal. Dichas variables fueron evaluadas mediante el auto reporte de los participantes en el cuestionario de FRM y de EdV, siendo en su caso la realización o no de alguna actividad física, o en el caso de las restantes variables el consumo o no de la sustancia.

\section{Procedimiento}

Los sujetos fueron encuestados en un consultorio anexo al consultorio de enfermería de un centro de salud privado de la ciudad de Mérida Yucatán. Se estableció un acuerdo formal con la coordinación del departamento de salud de dicho centro, y mediante entrevista con el director de dicha dependencia se 
Tabla 1

Estadísticos descriptivos de los factores de riesgo médico

\begin{tabular}{cccccc}
\hline Estadísticos & $\begin{array}{c}\text { PA Sistólica } \\
(\mathrm{n}=238)\end{array}$ & $\begin{array}{c}\text { PA Diastólica } \\
(\mathrm{n}=238)\end{array}$ & $\begin{array}{c}\text { Peso }(\mathrm{Kg}) \\
(\mathrm{n}=238)\end{array}$ & $\begin{array}{c}\text { Estatura }(m) \\
(\mathrm{n}=239)\end{array}$ & $\begin{array}{c}\text { IMC } \\
(\mathrm{n}=226)\end{array}$ \\
\hline & 117.10 & 74.45 & 70.32 & 1.57 & 28.52 \\
$\sigma$ & 15.11 & 10.11 & 13.37 & 0.09 & 4.43 \\
\hline
\end{tabular}

explicaron los objetivos, alcances y limitaciones del estudio. Los sujetos fueron encuestados voluntariamente y previo consentimiento informado por estudiantes de la Universidad Autónoma de Yucatán capacitados para solicitar el consentimiento de los pacientes, explicar los objetivos y limitaciones del estudio, así como para el levantamiento de los datos mediante la aplicación del cuestionario de FRM y de EdV, además, de la solicitud de mediciones antropométricas. La aplicación duró aproximadamente 20 minutos.

\section{Análisis de datos}

Para realizar los análisis estadísticos y obtener las tablas correspondientes se empleó el programa estadístico SPSS 16.0. Se verificaron los datos y se realizaron análisis para localizar inconsistencias en los datos, se recapturaron o eliminaron aquellos casos que lo requirieron debido a falta de información en el cuestionario de FRM y de EdV.

Para su análisis y para la presentación de resultados, las variables de los FRM, se organizaron en FRM discretos y continuos; y FHRM.

Se empleó la prueba estadística de $X i$ Cuadrada para el análisis de las variables categóricas, esta prueba estadística se emplea para conocer la asociación entre variables. También se empleo la prueba estadística no paramétrica de $U$ de Mann-Whitney, dicha prueba evalúa la hipótesis de que dos grupos provienen de muestras diferentes, en donde la variable independiente (VI) es una variable dicotómica y la, o las variables dependientes (VD), son variables continuas. En el caso de esta investigación la VI fue el diagnóstico de HTA y las VD fueron la PA sistólica, PA diastólica, peso, talla e IMC. Para la presentación de resultados se muestra la $n$ para indicar el número de datos válidos para cada variable.

\section{Resultados}

\section{Variables demográficas}

La muestra estuvo compuesta de 246 sujetos, la media de edad fue de 44.33 años (D.E.= 10.66). En cuanto al sexo, la muestra estuvo constituida por un $35.4 \%$ de hombres $(f=87)$ y un $64.6 \%$ de mujeres ( $f=159)$.

En cuanto a la presencia de HTA se observó que el $27.2 \%$ de la muestra reportó tener diagnóstico de HTA (Fr. =66) y el $72.8 \%$ reportó no tener diagnóstico de HTA $(F r .=177)$, comportándose esta variable conforme a lo esperado de acuerdo a las estadísticas locales y nacionales del SSA.

En lo que se refiere a los FRM, se pueden apreciar en la tabla 1, las descripciones de las medias, las desviaciones estándar y los FRM de las mediciones antropométricas; y en la tabla 2, las frecuencias y porcentajes de los FRM y FHRM medidos de forma categórica. 
Tabla 2

Estadísticos descriptivos de los factores de riesgo médico y factores hereditarios de riesgo médico

\begin{tabular}{llcccc}
\hline Factor & Variable & Presencia & \multicolumn{2}{c}{ Ausencia } & \\
\cline { 3 - 5 } & & $n$ & $F$ & $\%$ & $f$ \\
\hline \multirow{2}{*}{ FRM } & Control farmacológico & 87 & 62 & 71.3 & 25 \\
& Diabetes & 246 & 27 & 11.0 & 219 \\
& Disfunción renal & 246 & 32 & 13.0 & 214 \\
& Infarto & 246 & 4 & 1.6 & 242 \\
FHRM & Familia con hipertensión & 245 & 122 & 50.2 & 123 \\
& Familiar con disfunción cardiaca & 244 & 103 & 42.2 & 141 \\
& Familiar con diabetes & 245 & 155 & 63.3 & 90 \\
& Familiar con disfunción renal & 245 & 71 & 29.0 & 174 \\
\hline
\end{tabular}

En lo que respecta a los factores hereditarios, se pueden apreciar en la tabla 2, las frecuencias y porcentajes de patologías existentes en los familiares de primer grado. Llama la atención la paridad existente entre la frecuencia de familiares con y sin hipertensión, ya que esta frecuencia es de 122 y 123 respectivamente. También se observa una alta incidencia de familiares con diabetes, siendo ésta de 155 sujetos (63.3\%); en tanto que tan solo 90 $(36.7 \%)$ reportaron no haber tenido parientes de primer grado con diabetes.

\section{Tabla 3}

\section{Factores de estilo de vida}

\begin{tabular}{lcccc}
\hline Variable & Presencia & \multicolumn{2}{l}{ Ausencia } \\
\hline
\end{tabular}

Referente a los elementos de EdV que se consideraron para el estudio, se encontró una mayor presencia de no fumadores (88.6\%), así como de personas que no consumen sal sin probar previamente sus alimentos $(71.8 \%)$. Lo mismo ocurre con las personas que no ingieren alcohol, aunque de forma menos pronunciada $(62.2 \%)$. En tanto que la activación física (realizar ejercicio físico), mostró una presencia semejante (ver tabla 3).
En lo que se refiere a FRM asociadas a la HTA, se observó una asociación significativa entre los grupos formados por el diagnóstico de HTA, en la variable diabetes $\left(X^{2}{ }_{(1)}=14.05, p<.05\right.$; ver tabla 4), presentándose más diabéticos en el grupo de Ss con HTA (Fr. =16) que en el de Ss sin HTA (Fr. = 11; ver tabla 5).

\section{Tabla 4}

Análisis de Ji cuadrada de diferencias entre HTA y factores de riesgo médico

\begin{tabular}{lccc}
\hline Factor & $\mathbf{X}^{2}$ & $\boldsymbol{g l}$ & $\boldsymbol{p}$ \\
\hline Control farmacológico $(\mathrm{n}=87)$ & 68.44 & 1 & 0.00 \\
Diabetes $(\mathrm{n}=243)$ & 14.05 & 1 & 0.00 \\
Disfunción renal $(\mathrm{n}=243)$ & 0.595 & 1 & 0.44 \\
Disfunción cardiaca $(\mathrm{n}=8)$ & $--^{*--^{*}}$ & 1 & 1.00 \\
Infarto $(\mathrm{n}=243)$ & $-_{---^{*}}$ & 1 & 0.06 \\
\hline \multirow{2}{*}{ *Se usó el estadístico de Fisher. } & & &
\end{tabular}

Otra asociación que se presentó, fue en el consumo de un medicamento de control, lo cual era de esperarse debido a la naturaleza de la muestra $\left(X^{2}{ }_{(1)}=68.44, p<.05\right)$, ya que la mayoría de los participantes padecen HTA y consumen medicamentos de control, y no se encontraron participantes sin HTA que consumieran medicamento de control $(F r=0)$. Contra lo esperado, no se presentó asociación estadísticamente significativa entre el diagnóstico de HTA con disfunción cardiovascular $\left(X^{2}{ }_{(1)}=\right.$ $\mathrm{p}>.05)$, con padecimientos renal $\left(\chi_{(1)}^{2}=.595\right.$, $p>.05)$, ni con la variable presencia de infartos $\left(X_{(1)}^{2}=---, p>.05\right)$, (ver tabla 4). 
Tabla 5

Tabla de contingencia de Ss con y sin HTA en cuanto a diagnóstico o ausencia de diagnóstico de diabetes

\begin{tabular}{lcccc}
\hline \multicolumn{2}{c}{ Diabetes } & \multicolumn{3}{c}{ Total } \\
\hline & & Con diabetes & Sin diabetes \\
\hline $\begin{array}{l}\text { Diagnóstico } \\
\text { de HTA }\end{array}$ & Con HTA & 166 & 11 & 177 \\
& Sin HTA & 50 & 16 & 66 \\
Total & & & 216 & 27 \\
\hline
\end{tabular}

También se observó, que aunque en el grupo de personas con HTA hay más sujetos con diabetes, que en el grupo de Ss sin HTA; los Ss con HTA y con diabetes no reportan más familiares con antecedentes de diabetes que los Ss sin HTA $y$ con diabetes $\left(X_{(1)}^{2} p>.05\right)$ (ver tabla 6).
Tabla 6

Tabla de contingencia de Ss con diabetes con y sin HTA en cuanto a la presencia de familiares de 1 er grado con diabetes

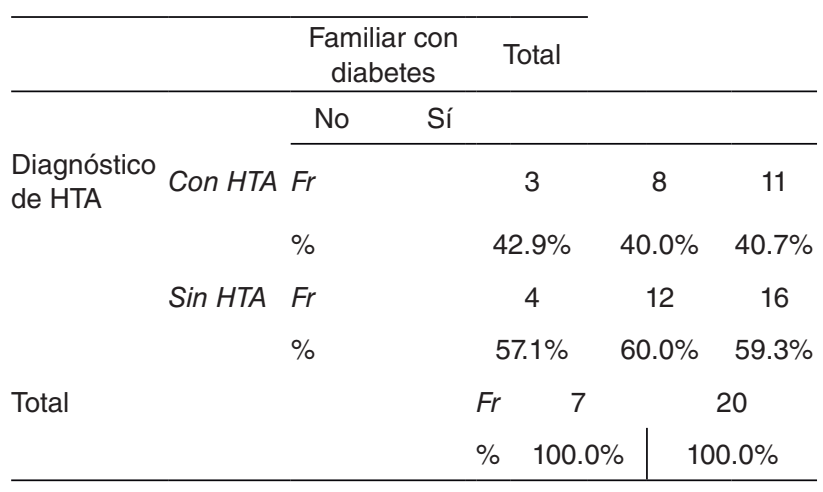

En lo que respecta a los factores de riesgo provenientes de las mediciones antropométricas, se pudieron apreciar diferencias entre los grupos de personas con y sin diagnóstico de HTA, en la presión arterial sistólica, presión arterial diastólica, peso e índice de masa corporal (IMC). Lo anterior se puede apreciar en los resultados del análisis de $U$ de Mann Whitney en la tabla 7.

\section{Tabla 7}

Diferencias mediante el análisis de $\mathrm{U}$ de Mann Whitney de factores de riesgo provenientes de mediciones antropométricas entre sujetos con y sin HTA

\begin{tabular}{lccccc}
\hline & PA Sistólica & PA Diastólica & Peso $(\mathrm{Kg})$ & Estatura $(\mathrm{m})$ & IMC \\
\hline U de Mann-Whitney & 2886.000 & 3132.500 & 3877.000 & 4914.000 & 3077.500 \\
Puntuación Z & -5.422 & -4.943 & -3.022 & -1.041 & -4.040 \\
p. (bilateral) & .000 & .000 & .003 & .298 & .000 \\
\hline
\end{tabular}

Se presentan también las medianas de las mismas variables en la tabla 8 , ya que resultan de más fácil comprensión en comparación con las medias de rangos presentadas por el análisis de $U$ de Mann-Whitney. 
Tabla 8

Medias de factores de riesgo médico provenientes de mediciones antropométricas por diagnóstico de hipertensión.

\begin{tabular}{lcccr}
\hline \multirow{2}{*}{ Variable } & & \multicolumn{2}{c}{$\begin{array}{c}\text { Con } \\
\text { diagnóstico } \\
\text { de HTA }\end{array}$} & $\begin{array}{c}\text { Sin } \\
\text { diagnóstico } \\
\text { de HTA }\end{array}$ \\
\hline PA Sistólica & 235 & 61 & 120.00 & 174 \\
\cline { 2 - 4 } PA Diastólica & 235 & 61 & 80.00 & 174 \\
Peso (Kg) & 236 & 61 & 72.5 & 175 \\
Estatura (m) & 237 & 63 & 1.56 & 174 \\
IMC & 224 & 59 & 29.97 & 165 \\
\hline
\end{tabular}

En cuanto a la presencia de padecimientos en los familiares de primer grado, no se encontraron asociaciones estadísticamente significativas entre los grupos de sujetos con HTA y los del grupo sin HTA en las variables padecimientos cardiacos $\left(\chi_{(1)}^{2}=2.758, p>.05\right)$, diabetes $\left(\chi_{(1)}^{2}=.337, p>.05\right)$ y padecimientos renales $\left(\chi_{(1)}^{2}=.024, p>.05\right.$; ver tabla 9 ).

Por el contrario, se encontraron asociaciones estadísticamente significativas entre el diagnóstico de HTA y la variable FHRM de familiar con hipertensión $\left(\chi_{(1)}^{2}=7.726, p<.05\right.$; ver tabla 9).

Tabla 9

Análisis de Ji cuadrada de asociación entre el diagnóstico de hipertensión arterial y los factores hereditarios de riesgo médico

\begin{tabular}{lccc}
\hline Factor & $\mathbf{X}^{2}$ & $\boldsymbol{g l}$ & $\boldsymbol{p}$ \\
\hline Familia con hipertensión & $\mathbf{7 . 7 2 6}$ & $\mathbf{1}$ &. $\mathbf{0 0 5}$ \\
Familiar con padecimiento cardiaco & 2.758 & 1 & .097 \\
Familiar con diabetes & .337 & 1 & .561 \\
Familiar con padecimiento renal & .024 & 1 & .877 \\
\hline
\end{tabular}

En la tabla 10 se puede apreciar que es mayor la frecuencia de personas con hipertensión y con familiar hipertenso ( $F r .=43 ; 65.2 \%)$, que sin él $(F r .=23,34.8 \%)$; en tanto que se comportó de manera semejante la frecuencia de personas sin hipertensión con familiar hipertenso ( $F r$. = $99 ; 55.9 \%)$ y sin él $(F r .=78 ; 44.1 \%)$.

\section{Tabla 10}

Tabla de contingencia de Ss con y sin HTA en cuanto a familiar de 1er grado con y sin HTA

\begin{tabular}{lrllll}
\hline & & \multicolumn{3}{c}{ Familiar con hipertensión } \\
\cline { 3 - 6 } & & & No & Sí & Total \\
\hline \multirow{3}{*}{ Diagnóstico de HTA } & No & Fr. & 99 & 78 & 177 \\
& $\%$ & $55.9 \%$ & $44.1 \%$ & $100 \%$ \\
& Sí & Fr. & 23 & 43 & 66 \\
Total & $\%$ & $34.8 \%$ & $65.2 \%$ & $100 \%$ \\
& & Fr. & 122 & 121 & 243 \\
& & $\%$ & $50.2 \%$ & $49.8 \%$ & $100 \%$ \\
\hline
\end{tabular}

En lo que se refiere a los indicadores de EdV, no se presentó asociación estadísticamente significativa entre el diagnóstico de HTA en ninguna de las variables (fumar $\left[X^{2}{ }_{(1)}=.000\right.$, $p>$.05], consumo de alcohol $\left[X_{(1)}^{2}=0.118, p>.05\right]$, activación física $\left.X_{(1)}^{2}=1.231, p>.05\right]$, consumo de sal $\left.\left[\chi_{(1)}^{2}=1.074, p>.05\right]\right)$.

\section{Discusión}

Contra lo esperado, los resultados encontrados cuestionan la inclusión de las disfunciones cardiovasculares, los padecimientos renales y la presencia de infartos, como factores de riesgo para la HTA, pues no se presentaron asociaciones estadísticamente significativas entre el diagnóstico de HTA y dichas variables. Lo mismo ocurrió en la asociación entre el diagnóstico de HTA y las variables de los FHRM en cuanto a familiares con padecimientos cardiacos, con diabetes y con padecimientos renales. 
Si se parte del hecho de que en la muestra estudiada, la incidencia de la HTA se comporta conforme a lo esperado de acuerdo a las estadísticas locales y nacionales de la SSA; llama la atención la paridad existente entre la frecuencia de familiares con y sin HTA en la muestra total. El desglose del dato cuestiona la contribución hereditaria en la HTA cuando se observa, que aunque es mayor la frecuencia de Ss con HTA y con familiar hipertenso que sin él, en el caso de los Ss sin HTA, la frecuencia de personas con familiar hipertenso y sin él se comporta de forma similar.

Con respecto a la diabetes, en esta muestra se observó una alta incidencia, presentándose más diabéticos en el grupo de Ss con HTA que en el de Ss sin HTA. A pesar de que la HTA y la diabetes están estrechamente relacionadas, no parece existir un nexo de carácter hereditario entre ellas, dado que en el grupo de personas con HTA hay más sujetos con diabetes que en el grupo de Ss sin HTA; los Ss con HTA y con diabetes no reportan más familiares con antecedentes de idabetes que los Ss sin HTA y con diabetes.
Los datos relativos a la influencia del EdV sobre la HTA, sugieren su participación solo como exacerbante y no como agente causal de la HTA, ya que los sujetos con y sin HTA respondieron igual en estas variables. También puede apreciarse en esta muestra una tendencia clara hacia el cuidado de la salud: hay mayor presencia de no fumadores, de Ss que no consumen sal sin probar previamente sus alimentos y que no ingieren alcohol. Ambos grupos realizan activación física con frecuencia semejante.

Las limitaciones de estudio serían que esta es una aproximación dentro de un proyecto mayor; los resultados aquí presentados muestran un primer análisis de la información obtenida. También es importante recalcar que la información obtenida ha sido recabada en su mayoría por autoreporte con los consiguientes cuestionamientos que de ello se deriva. Por último, son altamente relevantes para comprender los datos, las características particulares de la población entre la cual se realizó el estudio, ya que al ser una población en un sistema de salud privado con acceso inmediato a especialistas, diagnóstico y control farmacológico, permite un tratamiento oportuno e incluso prevención de patologías asociadas al diagnóstico de HTA. 


\section{Referencias}

Gasparotto, G., Silva, M., Bozza, R., Stabelini, N., Campos W., Bonfim, A.L. \& Costa, A. (2009). Physical activity and anthropometric indicators related to hypertension in women, Fitness and Performance, 8(5), 322-328.

Hernández, R. (2005). Tratamiento de Hipertensión arterial esencial estadio III, monodosis de nifedipino 30 mgvs atenolol $50 \mathrm{mg} /$ clortalidona12.5 $\mathrm{mg}$ Revista de la Facultad de Medicina UNAM; 48(4) 134-138. Recuperado el 16 de marzo de 2010, de http://www.ejournal. unam. $\mathrm{mx} / \mathrm{rfm} / \mathrm{no} 48-4 / \mathrm{RFM} 48403$.pdf.

Instituto Nacional de Salud Pública. (2006). Encuesta Nacional de Salud y Nutrición. Recuperado el 16 de marzo de 2010, de http://www.insp.mx/ensanut/.

Monotya, E. y Osorio, C. (2006). Variables psicológicas, relacionadas con hipertensión controlada y no controlada en un grupo de pacientes hipertensos adscritos a la IPS punto de Salud Robledo - Susalud EPS- en el año 2005. Recuperado el 15 de marzo de 2010, de http://www.susalud. com/memorias_prestadores/factores_hta_cont_no_cont.pdf

National Heart Lung and Blood Institute. (2008). ¿Qué es la presión arterial alta? Recuperado el 9 de noviembre de 2011, de http:// www.nhlbi.nih.gov/health-spanish/health-topics/temas/hbp/

Organización Mundial de la Salud [OMS]. (2010). Diabetes. Recuperado el 2 de octubre de 2011, de http://www.who.int/ mediacentre/factsheets/fs312/es/index.html

Organización Mundial de la Salud [OMS]. (2010). Enfermedades cardiovasculares. Recuperado el 2 de octubre de 2011, de http://www.who.int/mediacentre/factsheets/fs317/es/index. html

Rosas, M., Pastelín, G., Vargas-Alarcón, G., Martinez-Reding, J., Lomelí, C., Mendoza-Gonzales, C., et al. (2008). Guías clínicas para la detección, prevención, diagnóstico y tratamiento de hipertensión arterial sistémica en México. Arch Cardiol Mex; 78 (2) 5-57.

Silberberg, Ch. (2010). Enfermedad renal. Recuperado el 2 de octubre de 2011, de http://www.nlm.nih.gov/medlineplus/ spanish/ency/article/000457.htm

Velazquez-Monroy O., Rosas-Peralta M., Lara-Esqueda A., Pastelín-Hernandez G., Attie F.,Tapia-Conyer R. (2002). Arterial hypertension in Mexico: results of the National Health Survey 2000. Arch Cardiol Mex; 72, 71-84. Recuperado el 18 de marzo de 2010, de http://www.ncbi.nlm.nih.gov/ pubmed/11933703

World Healt Organization [WHO]. (2004). Comparative Quantification of Healt Risk, Geneva: WHO 